\title{
Predictive Fokker-Planck Modelling of Confined and Lost Fusion Alpha Particles in ITER
}

\author{
Victor Yavorskij • Victor Goloborod'ko • \\ Lars-Goran Eriksson • Vasili Kiptily • \\ Klaus Schoepf · Sergei E. Sharapov
}

Published online: 12 February 2015

(C) The Author(s) 2015. This article is published with open access at Springerlink.com

\begin{abstract}
The paper represents results of predictive 3D Fokker-Planck modelling of phase space distributions of fusion alpha particles for basic ITER scenarios (Polevoi et al. in J Plasma Fusion Res Ser 5:82, 2002). We simulate the poloidal profiles of alpha induced current as well as of the fusion power deposition to bulk plasma electrons. It is demonstrated that anisotropy of velocity distributions of alphas results in a rather strong alpha driven current that makes up about $10-15 \%$ of the equilibrium plasma current density in the 4th ITER scenario. We investigate the impact of the alpha driven current on the ITER magnetic configuration. In the 4 th scenario fusion alphas are shown to result in $\sim 15 \%$ enhancement of the rotational transform and in $\sim 11 \%$ enlargement of the Shafranov shift of magnetic flux surfaces. Also we evaluate the capability of gamma diagnostics of high-energy alphas in ITER and examine the collisional losses of fusion alpha particles.
\end{abstract}

Keywords Fusion alpha particles $\cdot$ Fokker-Planck equation · ITER · Distribution function · Plasma equilibrium

V. Yavorskij $(\bowtie) \cdot$ V. Goloborod'ko · K. Schoepf

Institute for Theoretical Physics, University of Innsbruck, Innsbruck, Austria

e-mail: Victor.Yavorskij@uibk.ac.at

V. Yavorskij · V. Goloborod'ko

Institute for Nuclear Research, Ukrainian Academy of Sciences,

Kiev, Ukraine

L.-G. Eriksson

European Commission, 1049 Brussels, Belgium

V. Kiptily · S. E. Sharapov

CCFE, Culham Science Centre, Abingdon, Oxfordshire, UK

\section{Introduction}

In comparison to the effect of charged fusion products (CFPs) in current tokamaks, CFPs in ITER are expected, due to the significantly enhanced fusion power, to have a stronger impact both on the plasma as well as on the first wall. Therefore development of plasma scenarios and research programs for ITER [2] requires a detailed modelling of fusion-born alphas confined in plasma as well as those lost to the first wall. Here we present results of predictive 3D Fokker-Planck modelling of fusion alphas for ITER Scenario 2 (standard H-mode, $\mathrm{I} / \mathrm{B}=15 \mathrm{MA} / 5.3 \mathrm{~T}$ ) and for Scenario 4 (steady-state, $\mathrm{I} / \mathrm{B}=9 \mathrm{MA} / 5.3 \mathrm{~T})[1]$.

The main attention is paid to the peculiarities of the velocity and poloidal distributions, $f_{\alpha}\left(\mathrm{v}_{11}, \mathrm{v}, R, Z\right)$, of confined alpha particles with energies exceeding hundreds of $\mathrm{keV}$. Using the distribution function $f_{\alpha}$ we calculate the poloidal profiles of alpha induced bootstrap current, $j_{\alpha}$, as well as of the fusion power deposition to electrons, $P_{\alpha e}$, and ions, $P_{\alpha i}$. The quantities $j_{\alpha}, P_{\alpha e}$ and $P_{\alpha i}$ characterize the CFPs effect on the plasma equilibrium and on the bulk plasma parameters depending on operation scenarios. To examine the capabilities of diagnostics of confined fast alpha-particles in ITER we evaluate also the $R, Z$ profiles of gamma-emission induced as a result of nuclear interactions of fusion alphas with $\mathrm{Be}$ and $\mathrm{C}$ impurity ions.

Finally we consider the diffusive loss of fusion alpha particles induced by Coulomb collisions and magnetic field inhomogeneity. We calculate the energy spectra and poloidal distributions of lost alphas as well as the maximum wall load induced by collisional loss of fusion alphas for basic ITER scenarios. 


\section{Modelling Results}

The simulation carried out is based on the 3D in constantsof-motion space Fokker-Planck approach previously used for modeling CFPs in TFTR [3-6] and JET [7, 8] as well as for NBI ions in JET $[9,10]$ and ITER [11]. Here we present the results of predictive modeling for fusion alphas in Scenario 2 (standard H-mode) and in Scenario 4 (steadystate operation). For these scenarios Fig. 1 displays the mid-plane profiles of safety factor in addition to the critical energy, $E_{\mathrm{cr}}$, Spitzer slowing-down time, $\tau_{\mathrm{se}}$ of alphas [12], DT fusion rate as well as of $Z_{\text {eff }}$. It is seen both effect of the radial width of alpha particle orbits as well as effect of Coulomb collisions are stronger for the Scenario 4. Note that rather high $(>20 \mathrm{keV})$, as compared to present day tokamaks, electron temperature in the ITER plasma core results in relatively high critical energy, $E_{\mathrm{cr}} \sim 0.9 \mathrm{MeV}$ (see Fig. 1b). As a result, in ITER plasma, collisions with the bulk plasma ions are essential even for partly thermalized alphas with $E \sim 1 \mathrm{MeV}$, while in plasmas of

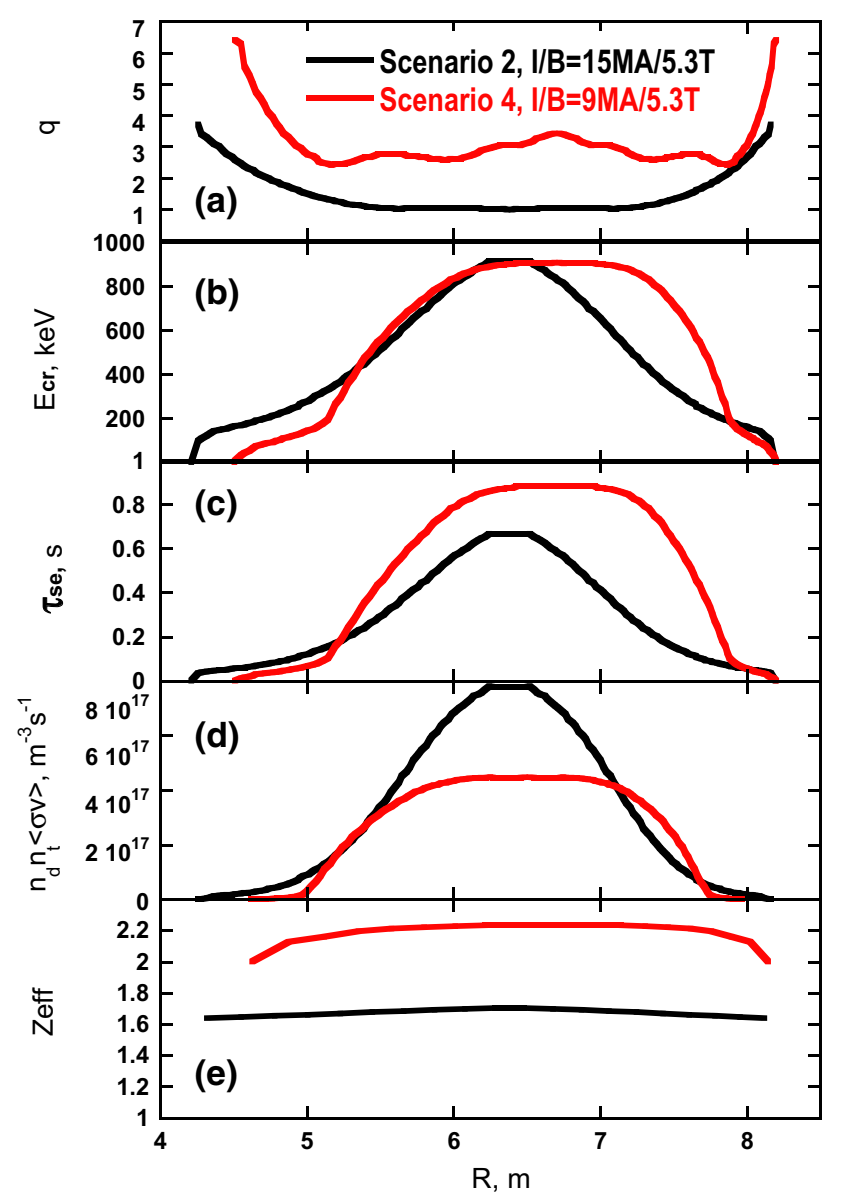

Fig. 1 The radial profiles of safety factor $(a)$, critical energy $\mathrm{E}_{\mathrm{cr}}(b)$, Spitzer slowing-down time $(c)$ of alpha particles, DT fusion rate $(d)$ and $\mathrm{Z}_{\mathrm{eff}}(e)$ [8] for 2nd and 4th ITER scenarios [1] present-day tokamaks the alpha-bulk ions collisions affect only the distribution function of well thermalized alphas with $E<<1 \mathrm{MeV}$. Correspondingly the collisional loss of fusion alphas, which is predominantly induced by pitchangle scattering on bulk plasma ions, should be more significant in ITER than in present-day tokamaks.

Note that our modelling supposes an MHD quiescent plasma, and correspondingly we neglect the effect of MHD induced transport of fast ions on the alpha particle confinement. Note in this context, that in ITER one may expect a weaker MHD impact on fast-ion transport than in presentday tokamaks. Reasons for that are, at least in case of MHD turbulence, the reduction of the $\rho_{*}$-parameter in ITER plasmas $\left(\rho_{*}=\mathrm{c}_{\mathrm{s}} /\left(\omega_{\mathrm{c}} a\right)\right.$ with $\mathrm{c}_{\mathrm{s}}$ denoting the sound speed, $\omega_{\mathrm{c}}$ the ion gyro-frequency and $a$ the plasma radius), as well as the control of MHD instabilities and potential suppression of turbulence by fusion alphas in ITER (see recent simulation studies in the frame of the FOM programme: http://www. fom.nl/live/english/about/annual_reports/artikel.pag?object number=257830).

\section{Distribution Function}

Obviously in ITER one may expect noticeable effect of collisional transport on the distribution function of even partly thermalized alphas. This is confirmed by Fig. 2 where compared are the contours of the modelled distribution function, $f_{\alpha}\left(R, \mathrm{v}_{11} / \mathrm{v}\right)$, of fusion alphas with energy $\mathrm{E}=3.5 \mathrm{MeV}$ and $\mathrm{E}=1.89 \mathrm{MeV}$ in the plasma mid-plane for both scenarios. It should be pointed out that the source term of fusion alphas is supposed to be mono-energetic. As expected the initial distribution of alphas in the reversed shear plasma (Scenario 4) is broader in R coordinate and is more anisotropic in longitudinal velocity as compared to those in standard 2nd Scenario. The anisotropy of alpha distributions is clearly seen in Fig. 2a, b where co-going $3.5 \mathrm{MeV}$ alphas are seen to be shifted to the low-B side as compared to the counter-going ones shifted to the high-B side. Moreover this shift is more pronounced in the case of 4th Scenario. Evidently the above-mentioned peculiarities of $R, \mathrm{v}_{11} / \mathrm{v}$ distributions are in correspondence with the orbit topology of fusion alphas produced in the plasma core. This is demonstrated by Fig. 3 where shown are 5 orbits of $3.5 \mathrm{MeV}$ alphas born at the magnetic axis $\left(R_{a x}=6.7 \mathrm{~m}\right.$, $\mathrm{Z}_{a x}=0.52 \mathrm{~m}$ ) of Scenario 4 plasma with an isotropic pitch-angle source distribution $\mathrm{v}_{\text {llax }} / \mathrm{v}=-\mathrm{v}_{\text {甲ax }} / \mathrm{v}=$ $\{-0.67,-0.32,0,0.32,0.67\}$. As expected, orbits of all the co-circulating particles, $0<\mathrm{v}_{\mathrm{llax}} / \mathrm{v}<1$, are seen to be contained at $R>R_{\mathrm{ax}}$, while orbits of counter-circulating particles, $-1<\mathrm{v}_{\text {llax }} / \mathrm{v}<-0.32$, are localised at $R<R_{\mathrm{ax}}$. As to toroidally trapped particles, $-0.32<\mathrm{v}_{\text {llax }} / \mathrm{v}<0$, their orbits belong to both $R>R_{\mathrm{ax}}$ as well as $R<R_{\mathrm{ax}}$ 

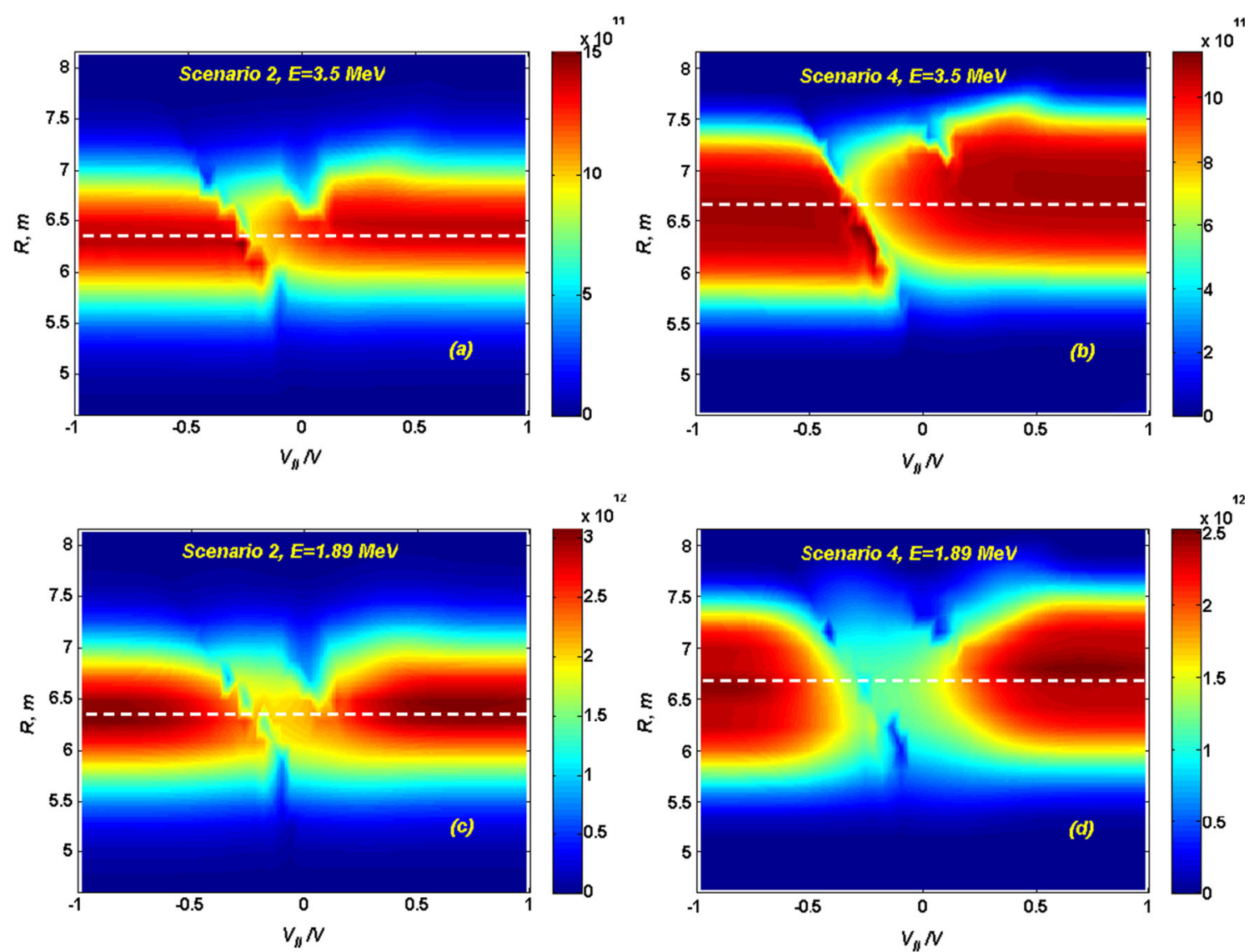

Fig. 2 The contours of the modelled distribution function, $\mathrm{f}_{\alpha}\left(\mathrm{R}, \mathrm{v}_{\mathrm{ll}} / \mathrm{v}\right.$ ), of fusion alphas with energy $\mathrm{E}=3.5 \mathrm{MeV}$ (plots a, b) and $\mathrm{E}=1.89 \mathrm{MeV}$ (plots $\mathbf{c}, \mathbf{d}$ ) in the plasma mid-plane for 2nd and 4th ITER scenarios

Fig. 3 Orbits of $3.5 \mathrm{MeV}$ alphas passing the paraxial region of plasma $\left(\mathrm{R}_{\mathrm{ax}}=6.7 \mathrm{~m}\right.$, $\left.\mathrm{Z}_{\mathrm{ax}}=0.52 \mathrm{~m}\right)$ at velocity $\mathrm{v}_{\mathrm{llax}} /$ $\mathrm{v}=\{-0.67$ (orbit A), -0.32 (orbit B), 0 (orbit C), 0.32 (orbit D), 0.67 (orbit E) \} in Scenario 4. Black parts of orbits correspond to $\mathrm{v}_{\varphi}>0$ and grey parts to $\mathrm{v}_{\varphi}<0$

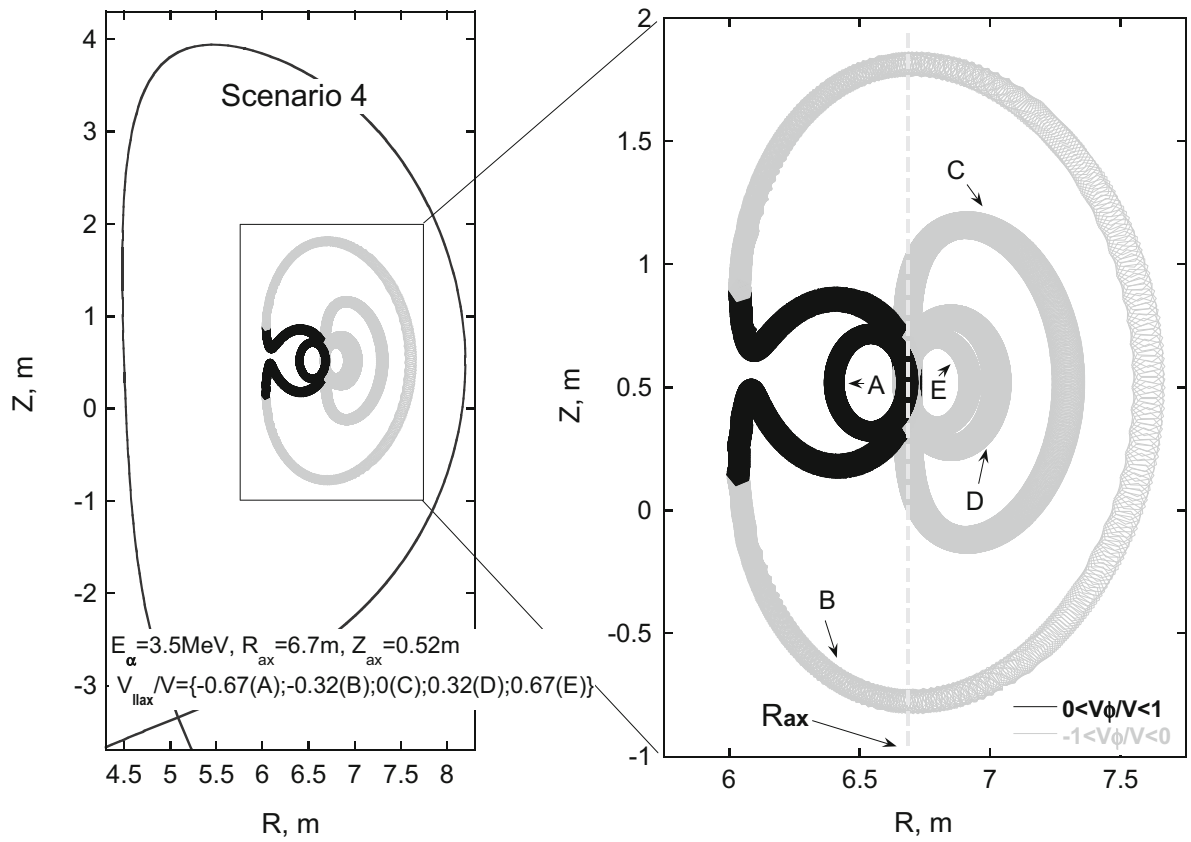


plasma area. Thus we conclude that majority of alphas from plasma core deviate to low-B $\left(R>R_{\mathrm{ax}}\right)$ plasma region where they are moving predominantly along the magnetic field. Contrary to the outer plasma area the majority of particles from high-B region are going against B. Consequently the distribution function of fusion alphas is substantially anisotropic even in the case of isotropic source term.

Figure $2 \mathrm{c}, \mathrm{d}$ represent mid-plane distributions $f_{\alpha}\left(R, \mathrm{v}_{11} / \mathrm{v}\right)$ of partly thermalized alphas with $E=1.89 \mathrm{MeV}$. The Coulomb collisions induced transport seen to redistribute the fast alphas both in the radial coordinate $R$ as well as in the longitudinal energy. However, the thermalized alphas are still substantially anisotropic over $\mathrm{v}_{11}$ and localised in the core of plasma. current $j_{\alpha}$ is identically directed with respect to the equilibrium plasma current in the vicinity of the outer part of mid-plane and is oppositely directed with respect to the equilibrium plasma current near the inner mid-plane of plasma (see also $[13,14]$ ). Note that the total alpha driven current $j_{\alpha \text { tot }}$, including that of alphas, $j_{\alpha}$, and also the electron reversed current, $j_{e},[15]$ can be represented as

$j_{\alpha \text { tot }}=j_{\alpha}+j_{e}, \quad j_{e}=-\frac{\left\langle j_{\alpha} / R\right\rangle_{F S}(1-G)}{R\left\langle R^{-2}\right\rangle_{F S}} \frac{Z_{\alpha}}{Z_{e f f}}$,

where $G$ represents the trapped electron correction to the Ohkawa current [16], $Z_{\text {eff }}$ is the effective charge number (see Fig. 1d) and $\langle\ldots\rangle_{F S}$ means the flux surface average (see Eq. (21) of Appendix). Using here expression for $G$ obtained in Ref. [17] we arrive at

$$
1-G \cong \frac{1.414+Z_{\text {eff }}+x\left(0.447+Z_{\text {eff }}\right)}{1.414+Z_{\text {eff }}+x\left(0.754 Z_{\text {eff }}^{-1}+2.657+2 Z_{\text {eff }}\right)+x^{2}\left(0.384 Z_{\text {eff }}^{-1}+1.243+Z_{\text {eff }}\right)}
$$

\section{Alpha-driven Current}

Due to anisotropy in longitudinal velocity the fusion alphas can generate a substantial longitudinal current $j_{\alpha}$ in the plasma. Figure 4 displays the $R, Z$ distribution of the density of the current driven by fusion alphas in ITER plasmas for 2nd (LHS) and 4th (RHS) Scenarios. Due to the excess of the co-going alphas in the low-B side and of the counter-going ones in the high-B side (see Fig. 3) the where $x=\eta_{t} / \eta_{t}\left(1-\eta_{t}\right)$ and $\eta_{t} \sim(r / R)^{1 / 2}$ stands for the fraction of trapped electrons on the flux surface with radius $r$. Using the analytical model of tokamak magnetic field with noncircular flux surfaces [18] for $\eta_{t}$, applicable for ITER magnetic configuration, we obtain [see Eq. (20)]

$\eta_{t}(r)=\left\langle\sqrt{1-\frac{R_{\min }}{R}}\right\rangle_{F S}$
Fig. 4 Modelled R,Z profiles of the density of alpha driven current in $\mathrm{Am}^{-2}$ for the 2nd (left) and 4th (right) ITER scenario
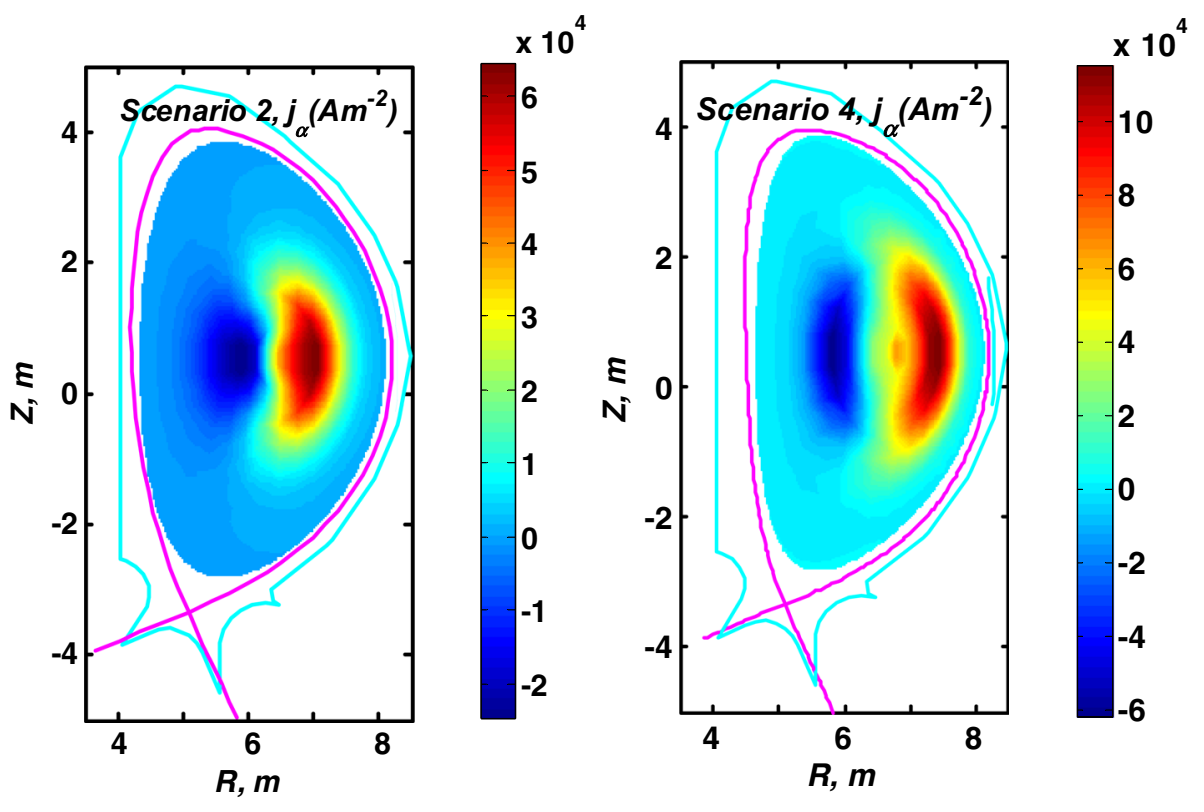
Fig. 5 Mid-plane profiles of the modelled density of alpha driven current for the 2 nd (a) and 4th (b) ITER scenario (a)

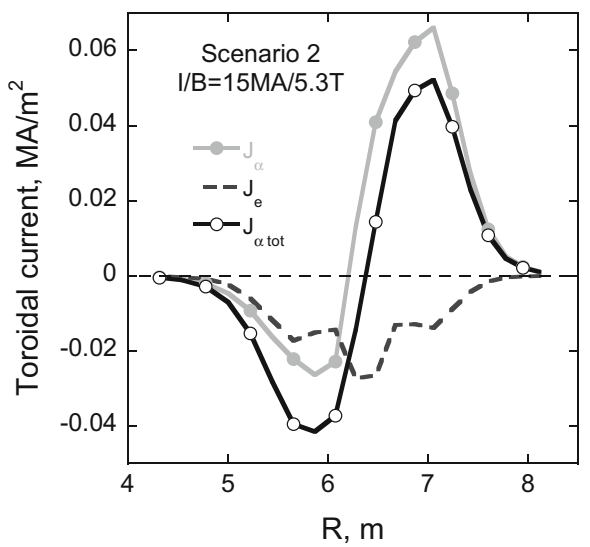

(b)

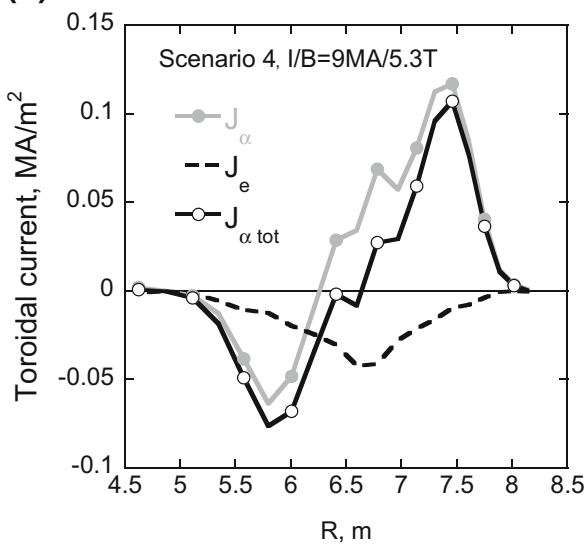

where $R_{\min }$ is the minimum $R$ along the orbit. Figure 5 displays the midplane profiles of the modelled currents of $j_{\alpha}, j_{e}$ and $j_{\alpha}$ tot for the 2 nd and 4th ITER scenarios. It is seen that electron current $j_{e}$ predominantly suppresses the poloidally symmetric component of $j_{\alpha}$ and makes the resultant profile of $j_{\alpha \text { tot }}$ close to completely asymmetric one in poloidal angle with $\left\langle j_{\alpha} \text { tot }\right\rangle_{F S}$ much smaller then $<j_{\alpha}>_{F S}$ and correspondingly with $\max \mid j_{\alpha}$ tot $\left(R<R_{\mathrm{ax}}\right)$ $\mathrm{I} \approx \max \left|j_{\alpha \text { tot }}\left(R>R_{\mathrm{ax}}\right)\right|$. It is important that alpha driven current in 4th Scenario is about two times higher as compared to those in 2nd Scenario.

From the point of view of the alpha effect on the plasma equilibrium, important is the ratio of the alpha driven current $j_{\alpha}$ tot with respect to the total plasma current $j_{e q}$ [1] neglecting the fusion alpha contribution.

Figure 6 compares the densities of $j_{\alpha t o t}\left(R, Z_{a x}\right), j_{e q}$ $\left(R, Z_{a x}\right)$ and of the total plasma current including fusion alpha contribution (solid black lines) in the plasma midplane for 2 nd and 4 th Scenario. It is seen that alpha driven current can comprise a significant part of plasma current and in the case of 4 th Scenario can make up to $10-15 \%$ of the equilibrium plasma current density without $j_{\alpha}$ tot as calculated by ASTRA [1]. Therefore alpha current can noticeably affect the plasma equilibrium especially in the case of reversed shear plasma in 4th Scenario. Note that reversed shear induced enhancement of alpha driven current in ITER is in agreement with the current hole enlargement of $j_{\alpha}$ observed in Monte-Carlo modelling of paper [13]. Important from the point of view of the alpha impact on tokamak plasma is the value of total alpha driven current, $I_{\alpha \text { tot }}=I_{\alpha}+I_{e}$, where

$$
\begin{aligned}
I_{\alpha} & =\iint d R d Z j_{\alpha}=\int d r V^{\prime}\left\langle j_{\alpha} / R\right\rangle_{F S}, \\
I_{e} & =\iint d R d Z j_{e}=-\int d r V^{\prime}\left\langle j_{\alpha} / R\right\rangle_{F S}(1-G) \frac{Z_{\alpha}}{Z_{e f f}}, \\
V^{\prime} & =\int_{0}^{2 \pi} d \chi Y R^{2}
\end{aligned}
$$

Table 1 represents the calculated values of current induced by alphas, $I_{\alpha}$, reversed electron current, $I_{e}$, as well as total alpha driven current, $I_{\alpha \text { tot }}$, for 2 nd and 4th Scenario.
Fig. 6 Mid-plane profiles of the modelled density of alpha driven current as compared to the total plasma current $j_{\mathrm{eq}}$ [1] for the 2nd (a) and 4th (b) ITER scenario (a)

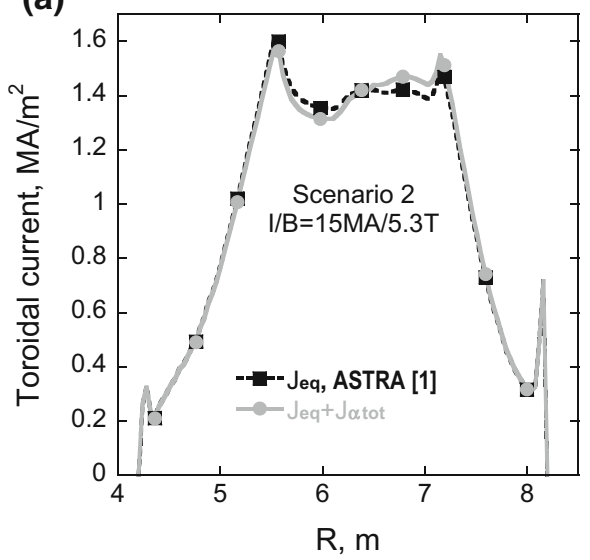

(b)

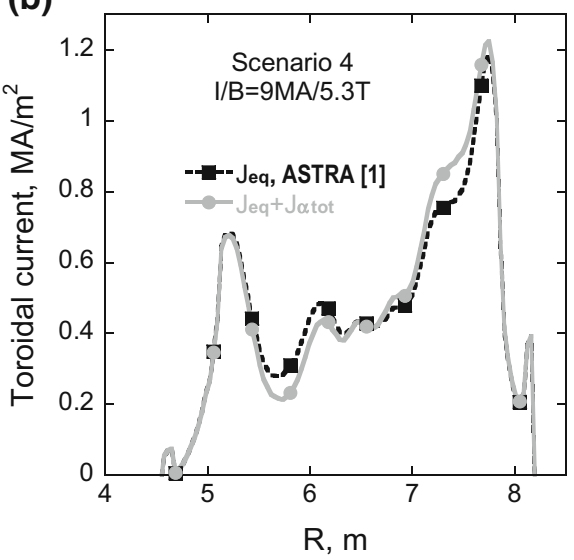


Table 1 Alpha driven current

\begin{tabular}{llll}
\hline Current components & $I_{\alpha}, \mathrm{MA}$ & $I_{e}, \mathrm{MA}$ & $I_{\alpha \text { tot }}, \mathrm{MA}$ \\
\hline Scenario 2 & 0.696 & -0.521 & 0.175 \\
Scenario 4 & 1.24 & -0.758 & 0.482 \\
\hline
\end{tabular}

As expected, electrons compensate substantial part of the alpha induced current $(\sim 0.7 \mathrm{MA}$ in 2 nd scenario and 1.2MA in 4th scenario). As a result the total current driven by fusion alphas is rather low contributing to full toroidal current only $<0.2 \mathrm{MA}$ and $<0.5 \mathrm{MA}$ in 2 nd and 4 th scenarios respectively. Evidently the alpha driven current will result in small reduction of safety factor $\sim I_{\alpha}$ tot $/ I$, which amounts $\sim 5 \%$ in 4 th scenario and only $\sim 1 \%$ in 2 nd scenario.

Alpha-particle Impact on the Plasma Equilibrium and On the Bulk Plasma Parameters

In spite of the rather low effect of total current driven by fusion alphas, $j_{\alpha}$ may result in a consequential redistribution of the toroidal current in the plasma core and thereby alter the plasma equilibrium in addition to $I_{\alpha}$ tot induced enhancement of rotational transform (reduction of safety factor). To demonstrate this we use the code HELENA [19] to evaluate the alpha current effect on the safety factor and the Shafranov shift. Note that with reasonable accuracy the current density $j_{\alpha}$ tot $(R, Z)$ can be approximated by the equilibrium-like profile

$\hat{j}_{\alpha t o t}=\Gamma_{\alpha}(\psi) \frac{R_{c}}{R}+\Pi_{\alpha}(\psi)\left(\frac{R}{R_{c}}-\frac{R_{c}}{R}\right)$,

where $R_{\mathrm{c}}$ denotes the major radius of the plasma centre ( $=6.2 \mathrm{~m}$ in 2 nd and $6.35 \mathrm{~m}$ in 4th Scenario), $\psi$ is an unperturbed poloidal flux (neglecting $j_{\alpha \text { tot }}$ ). According to our estimations the deviation of the approximate alpha driven current from exact one, $\delta j=j_{\alpha, t o t}-\hat{j}_{\alpha, t o t}$, is rather low. Thus $-0.6 \mathrm{kA} / \mathrm{m}^{2}<\delta j<0.5 \mathrm{kA} / \mathrm{m}^{2}$ for 2 nd Scenario and $-3 \mathrm{kA} / \mathrm{m}^{2}<\delta j<2.5 \mathrm{kA} / \mathrm{m}^{2}$ for 4 th Scenario Fig. 7 displays the profiles of $\Pi(\psi)$ and $\Gamma(\psi)$ in the case of unperturbed equilibrium configuration [1] and those accounting for $\Gamma_{\alpha}(\psi)$ and $\Pi_{\alpha}(\psi)$ in 2nd and 4th ITER scenarios. It is seen that alpha particles change predominantly $\Pi(\psi)$ while $\Gamma(\psi)$ is practically unaffected. Therefore, taking into account $j=j_{e q}[R, \Gamma(\psi), \Pi(\psi)]$ resulting from Eq. (5), we conclude that variation of toroidal current $\Delta j_{e q} / j_{e q}$ induced by $j_{\alpha \text { tot }}$ can be estimated as

$\frac{\Delta j_{e q}}{j_{e q}} \simeq 2 \frac{r}{R_{c}} \frac{\Delta \Pi}{\Gamma} \simeq 2 \frac{r}{R_{c}} \frac{\Pi_{\alpha}}{\Gamma}$.

Using expressions of Eq. (6) and profiles $\Pi(\psi), \Gamma(\psi)$ shown in Fig. 7 we conclude that $\Delta j_{e q} / j_{e q}<0.03$ for 2 nd Scenario and $<0.15-0.20$ for 4 th Scenario.

To evaluate quantitatively the alpha impact on magnetic configuration of ITER we compare HELENA calculations of equilibrium in case of unperturbed toroidal current $j=j_{e q}\left[R, \Gamma_{e q}(\psi), \Pi_{e q}(\psi)\right]$ and in the case when alpha current is included, i.e. when $j=j_{e q}\left[R, \Gamma_{e q}(\psi)+\Gamma_{\alpha}\right.$ $\left.(\psi), \Pi_{e q}(\psi)+\Pi_{\alpha}(\psi)\right]$. Effect of alpha current on the safety factor and the Shafranov shift is illustrated in Figs. 8 and 9. For the 4th ITER scenario it is seen that the alpha driven current reduces the safety factor in the plasma core by about $15 \%$ and enlarges there the Shafranov shift by $\sim 11 \%$.

The present study demonstrates that fusion alphas are expected to induce an additional rotational transform of the magnetic field lines in reactor size tokamak plasmas. In reversed shear plasma scenarios the impact of the alpha driven current appears to be greater. While in the ITER steady state scenario alpha particles induce a $15 \%$ reduction of the safety factor $q$ in the core area, in the 2nd ITER Scenario with positive shear the reduction of $q$ in the core
Fig. 7 Profiles of $\Pi(\psi)$ and $\Gamma(\psi)$ in the case of unperturbed equilibrium ITER configuration [1] (broken lines) and those disturbed by alpha driven current $\hat{j}_{\alpha \text { tot }}$ [see Eq. (5)] for 2 nd (left) and 4th (right) Scenarios
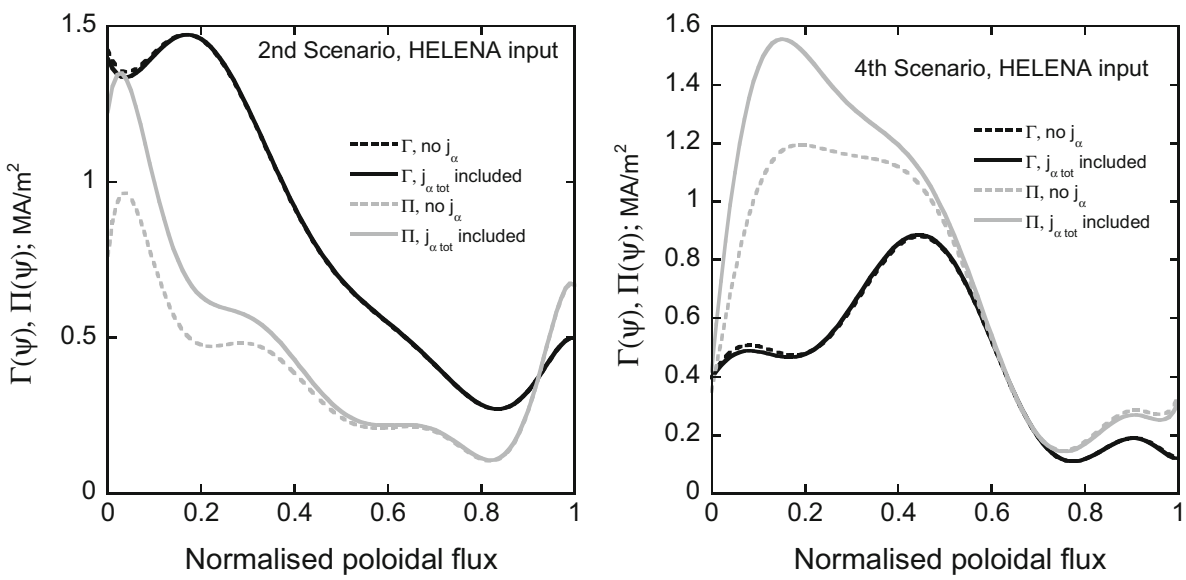


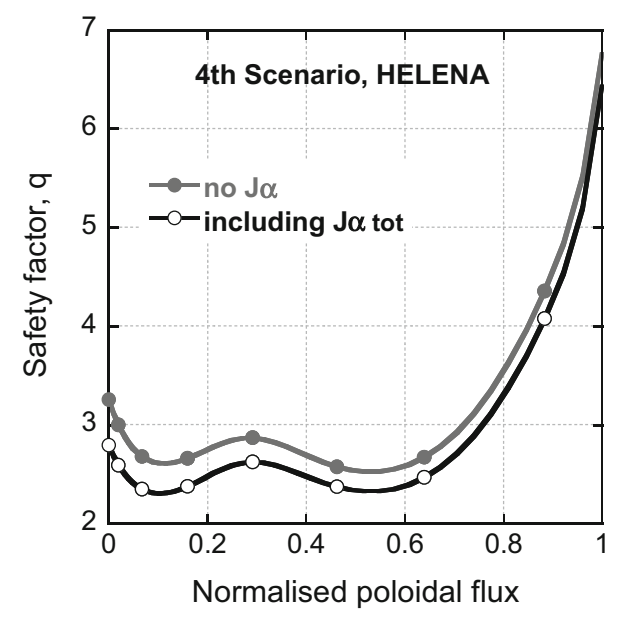

Fig. 8 Radial profiles of the modelled safety factor neglecting (grey curve) and accounting for the alpha driven current (black curve)

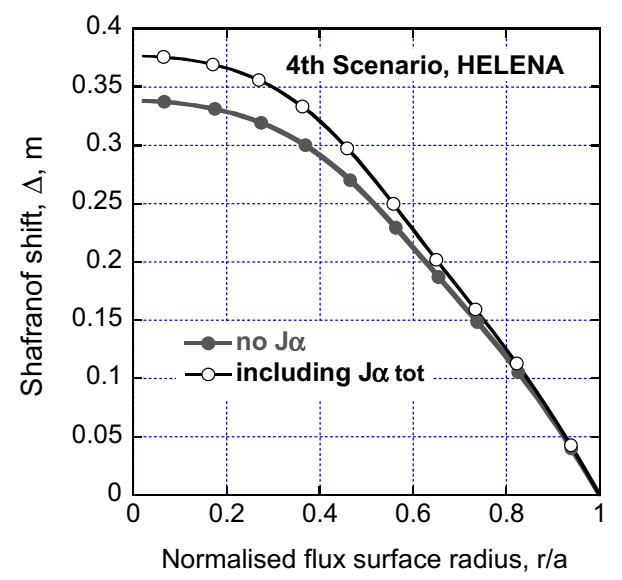

Fig. 9 Radial profiles of the modelled Shafranov shift neglecting (grey curve) and accounting for the alpha driven current (black curve)

is $<3 \%$ according to our calculations. Nevertheless, also such an alteration may reduce the core safety factor, which in Scenario 2 is only 1.02-1.04 [1], to a value below 1, the crucial value for plasma stability [20]. It is noted that, in spite of the low intensity of the total current driven by fusion alphas, $j_{\alpha}$ can play a role of a seed current for the bootstrap tokamak reactor. Evidently, the development of advanced plasma scenarios and research programs for ITER and future tokamak reactors should account for the effects of currents driven by fusion alphas.

Also significant for plasma equilibrium is a partial contribution of fusion alphas to the plasma pressure as well as the anisotropy of the latter. In spite of the relatively low density of energetic alphas as compared to those of bulk plasma components $\left(N_{\alpha}<8.9 \cdot 10^{17} \mathrm{~m}^{-3}<0.8 \% n_{\mathrm{e}}\right.$ in 2 nd scenario and $<6.2 \cdot 10^{17} \mathrm{~m}^{-3}<0.8 \% n_{\mathrm{e}}$ in 4 th one as seen in Fig. 10), their contribution to plasma beta can exceed $10 \%$ [1] due to the high energy of alpha particles.
To evaluate the anisotropy of the alpha particle pressure, we consider transverse, $p_{\alpha \perp}$, and longitudinal, $p_{\alpha \mathrm{ll}}$, components of the pressure tensor [21]

$\overleftrightarrow{p}_{\alpha}=\left(p_{\alpha \|}-p_{\alpha \perp}\right) \mathbf{b b}+p_{\alpha \perp} \overleftrightarrow{\mathbf{I}}$,

where $p_{\alpha \perp}$ and $p_{\alpha \| l}$ are given by

$p_{\alpha \perp}=\frac{m_{\alpha}}{2} \int v_{\perp}^{2} f_{\alpha} d \mathbf{v}, \quad p_{\alpha \|}=m_{\alpha} \int v_{\|}^{2} f_{\alpha} d \mathbf{v}$,

$\mathbf{b}=\mathbf{B} / \mathbf{B}, \mathbf{B}$ is the magnetic field and $\overleftrightarrow{\mathbf{I}}$ is the unit dyad. In expressions (8) we take into account the gyrotropic structure of the alpha particle distribution function, $f_{\alpha}(\mathbf{v})=f_{\alpha}\left(\mathrm{v}_{\|}, \mathbf{v}_{\perp}\right)$, as well as the smallness of the averaged longitudinal velocity, i.e.

$$
\left(\int v_{\|} f_{\alpha} d \mathbf{v}\right)^{2}<10^{-2} \int f_{\alpha} d \mathbf{v} \int v_{\|}^{2} f_{\alpha} d \mathbf{v}
$$

Figure 11 demonstrates the mid-plane profiles of $p_{\text {all }}$ and $p_{\alpha \perp}$ of energetic alphas $(E>320 \mathrm{keV})$ for Scenario 4 .

Shown in this figure is also a "mean" pressure $p_{\alpha}=$ $\operatorname{tr}\left(\overleftrightarrow{p}_{\alpha}\right) / 3=\left(p_{\alpha \|}+2 p_{\alpha \perp}\right) / 3$ corresponding to the alpha particle contribution to total plasma pressure. It is seen that $\left(p_{\alpha 11}-p_{\alpha \perp}\right) / p_{\alpha 11}$ can exceed $40-45 \%$ at low-B side thus introducing substantial $(\sim 4-5 \%)$ anisotropy to total pressure of ITER plasma.

Important in this context are also the electron and ion power deposition profiles, $P_{\alpha \mathrm{e}}$ and $P_{\alpha \mathrm{i}}$.

Figure 12 compares the poloidal profiles of alpha power deposited to electrons for 2 nd and 4 th scenario. As expected, magnetic reversed shear in 4th scenario results in broadening and in reduction of $P_{\alpha \mathrm{e}}$ as compared to those in 2nd scenario. Powers deposited to the bulk plasma ions $P_{\alpha \mathrm{d}}$ and $P_{\alpha \mathrm{t}}$ are small and comprise, respectively, $<20$ and $15 \%$ of $P_{\alpha \mathrm{e}}$.

\section{Collisional Loss of DT Alphas}

For evaluation of the fusion alpha impact on the first wall of ITER important is the predictive modelling of the alpha loss. In spite of the low level of the first orbit losses in ITER the collisional ones are significant. Figure 13 represents the fraction of fusion alphas, $L(E)$, lost at energies greater than $E$ as well as the energy spectra of these particles, $E d L(E) / d E$, for the 2nd and 4th ITER scenario. It is seen that alphas with energy ranges $3 \mathrm{MeV}<$ $E<3.5 \mathrm{MeV}$ and $0.5 \mathrm{MeV}<E<1 \mathrm{MeV}$ contributes predominantly to collisional losses. It is important to note that at rather high energies $E>1 \mathrm{MeV}$ alpha losses for 2nd scenario (with small radial excursions of alphas) exceed those for 4 th scenario (with large radial excursions of 
Fig. 10 Modelled R,Z profiles of the density of fusion alphas $(320 \mathrm{keV}<\mathrm{E}<3.5 \mathrm{MeV})$ in $\mathrm{m}^{-3}$ for the 2 nd $($ left $)$ and 4 th (right) ITER scenario

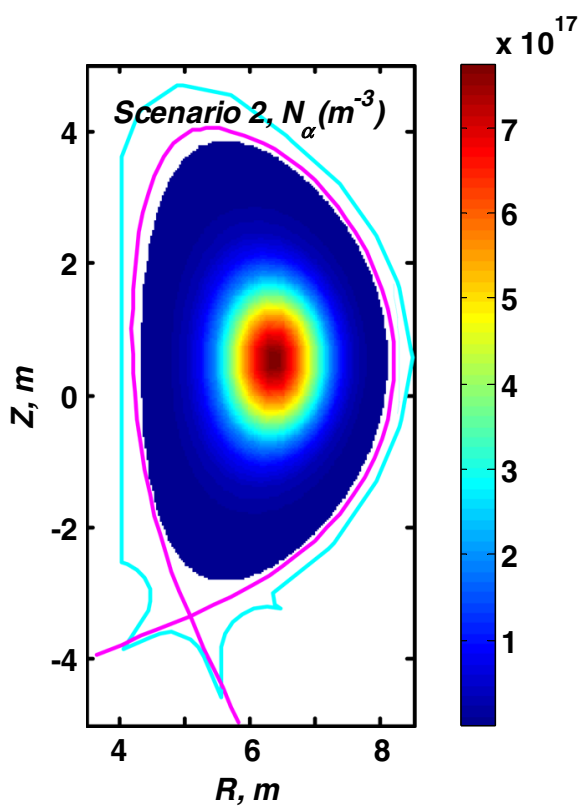

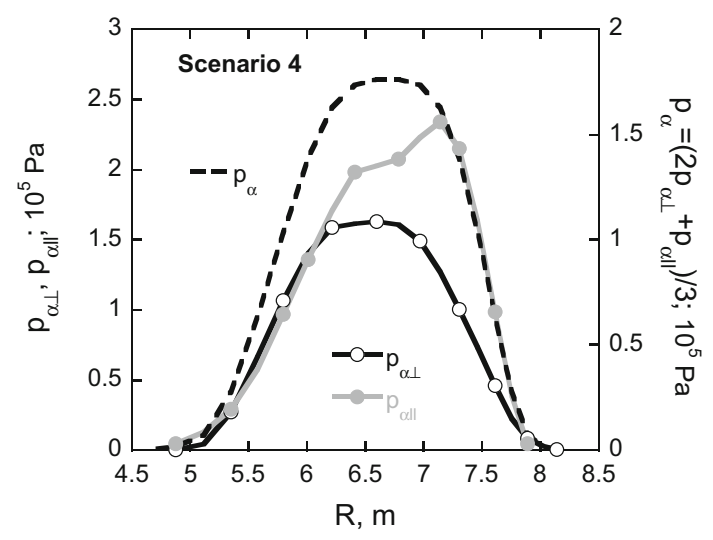

Fig. 11 Mid-plane profiles of the modelled components of pressure $\mathrm{p}_{\alpha \mathrm{ll}}, \mathrm{p}_{\alpha \perp}$ and "mean" pressure $\mathrm{p}_{\alpha}=\left(\mathrm{p}_{\alpha \mathrm{ll}}+2 \mathrm{p}_{\alpha \perp}\right) / 3$ of alphas with $\mathrm{E}>320 \mathrm{keV}$ for the 4th ITER scenario

alphas). This is due to extremely low level of fusion production at the plasma periphery $(r>0.7 \mathrm{a})$ in the case of 4th scenario [1] shown in Fig. 1d. However, in wider energy range $0.3 \mathrm{MeV}<E<3.5 \mathrm{MeV}$ the total collisional loss fraction of alphas for the 4th scenario is about $31 \%$ contrary to only $24 \%$ loss fraction for the 2 nd scenario. Note that enhanced collisional loss of partly thermalised fusion alphas with energy $E<1.7 \mathrm{MeV}$ were obtained in current hole JET plasma [8] as well as predicted in ITER in presence of TF ripples [22, 23].

\section{$\gamma$-Emission Induced by Fusion Alphas}

Finally Fig. 14 demonstrates the mid-plane profiles of partly thermalized alphas with energy $E>1.9 \mathrm{MeV}$ and profiles of $\gamma$-emission rates $R^{\gamma}$ from ${ }^{9} \mathrm{Be}(\alpha, n \gamma){ }^{12} \mathrm{C}$ reactions induced by alphas with energies $E>1.7 \mathrm{MeV}$, given by

$$
\begin{aligned}
R_{\alpha B e}^{\gamma}(R, Z) & =n_{B e}(R, Z) 2 \pi \int v^{3} f_{\alpha}(R, Z, E, \xi) \sigma_{\alpha B e}^{\gamma}(E) d \xi d v, \xi \\
& =\mathrm{v}_{\|} / \mathrm{v} .
\end{aligned}
$$

In Eq. (9) $n_{B e}$ represents the density of the Be impurity, the distribution function $f_{\alpha}(R, Z, E, \xi)$ refers to fusion alphas and $\sigma_{\alpha B e}^{\gamma}$ denotes the cross-section of ${ }^{9} \mathrm{Be}(\alpha, n \gamma)^{12} \mathrm{C}$ reaction. It is seen that for both scenarios collisional transport results in essential broadening of the mid-plane profiles of partly thermalized alphas compared to the initial profiles of alphas at birth energy. The fact that the $\gamma$-ray emission profiles are almost identical to the density profiles of partly thermalized alphas illustrates the value of this particular diagnostic technique.

\section{Summary}

Present predictive Fokker-Planck modelling of fusion alphas in ITER demonstrates the prominent sensitivity of their phase space distributions to the plasma scenarios. Thus significant dissimilarity of distributions over $R, Z$ spatial coordinates and over the longitudinal energy is observed for the 2nd and 4th ITER scenario. Particularly the longitudinal anisotropy of alpha distributions in 4th scenario results in rather strong alpha driven current that consists up to $10-15 \%$ of the equilibrium plasma current density. Consequently fusion alphas are expected to induce an additional rotational transform of the magnetic field lines in reactor 
Fig. 12 Modelled R,Z profiles of the electron power deposition profiles, $P_{\alpha \mathrm{e}}$ in $\mathrm{Wm}^{-3}$ for the 2nd (left) and 4th (right) ITER scenario
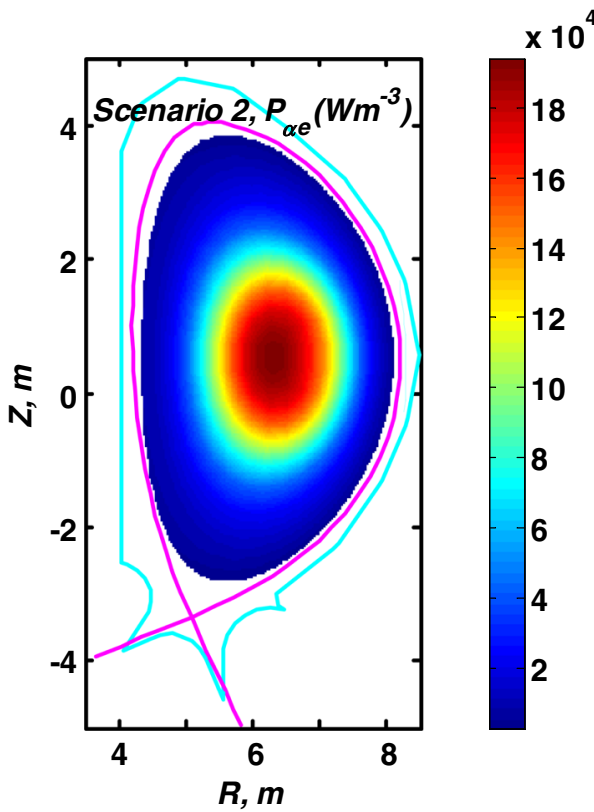

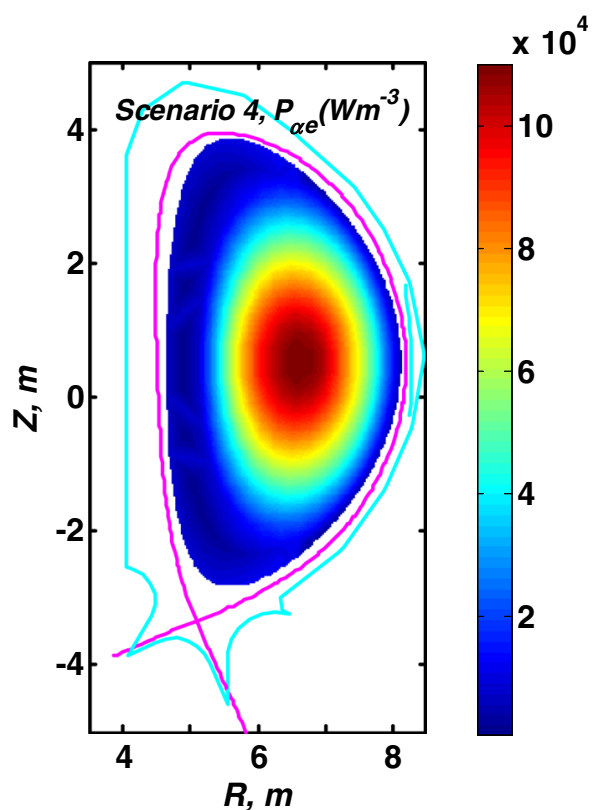

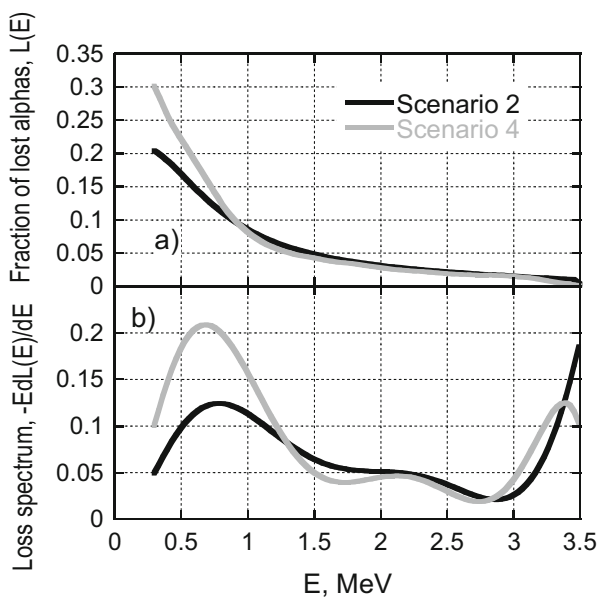

Fig. 13 Modelled alpha particle loss fraction (left) and energy spectra of lost alphas (right) for the 2nd and 4th ITER scenario size tokamak plasmas. In reversed shear plasma scenarios the impact of the alpha driven current appears to be greater. While in the ITER steady state scenario alpha particles induce a $15 \%$ reduction of the safety factor $q$ in the core area, in the 2nd ITER Scenario with positive shear the reduction of $q$ in the core is $<3 \%$ according to our calculations. Nevertheless, such an alteration may reduce the core safety factor, which in Scenario 2 is only 1.02-1.04 [1], to a value below 1 , the crucial value for plasma stability [20]. Alpha driven current shown also to result in essential $(\sim 11 \%$ in 4th Scenario) enlargement of the Shafranov shift. It is noted that, in spite of the low intensity of the total current driven by fusion alphas, $j_{\alpha}$ can play a role of a seed current for the bootstrap tokamak reactor. Evidently, the development of advanced plasma scenarios and research programs for ITER and future tokamak reactors should account for the effects of currents driven by fusion alphas.
Fig. 14 Modelled profiles of the $\gamma$-emission rates from ${ }^{9} \mathrm{Be}(\alpha, \mathrm{n} \gamma){ }^{12} \mathrm{C}$ reactions (broken lines), density of partly thermalized alphas

$\mathrm{N}_{\alpha}(\mathrm{E}>1.9 \mathrm{MeV})$ - (black solid lines) and $\mathrm{N}_{\alpha}(\mathrm{E}=3.5 \mathrm{MeV})-($ grey solid lines) for the 2nd scenario (left) and 4th scenario (right)
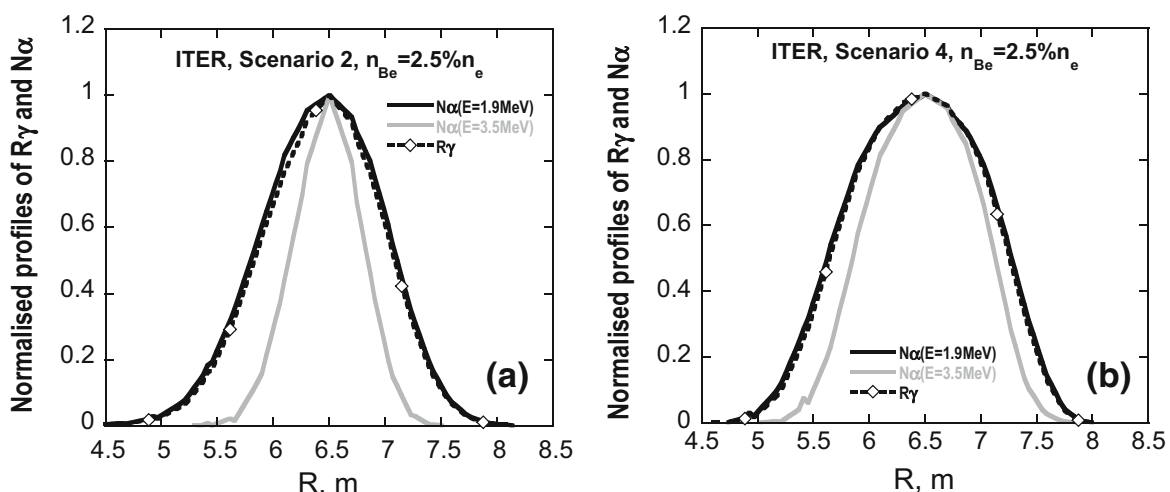
Anisotropy of the alpha particle pressure $\left(p_{\alpha \| l}-p_{\alpha \perp}\right) /$ $p_{\alpha \perp}$ can exceed 40-45\% at low-B side of plasma in Scenario 4 thus introducing substantial $(\sim 4-5 \%)$ anisotropy to total plasma pressure.

The poloidal profiles of alpha density as well as of the fusion power deposition to electrons and ions are found to be profoundly sensitive to operational scenarios. Powers deposited to the bulk plasma ions are small and consist $<1 / 3$ of those deposited to electrons. Radial profiles of $\gamma-$ emission rates from ${ }^{9} \mathrm{Be}(\alpha, n \gamma){ }^{12} \mathrm{C}$ reactions induced by fusion alphas are shown to be consistent with the profiles of partly thermalized alphas with energy $E>1.9 \mathrm{MeV}$.

Finally modelling performed demonstrated that Coulomb collisions result in a substantial (about 25-30 \%) loss of partly thermalized fusion alphas $(E>0.32 \mathrm{MeV})$ with the energy spectra of lost alphas been sensitive to the plasma scenarios.

Acknowledgments This work has been carried out within the framework of the EUROfusion Consortium and has received funding from the Euratom research and training programme 2014-2018 under grant agreement no. 633053. It was supported also by RCUK Energy Programme [grant no. EP/I501045]. The views and opinions expressed herein do not necessarily reflect those of the European Commission. Authors would like to thank Prof. Ya. Kolesnichenko, Dr. V. Drozdov and Dr. K. McClements for fruitful discussions on alpha driven current and alpha impact on plasma equilibrium.

Open Access This article is distributed under the terms of the Creative Commons Attribution License which permits any use, distribution, and reproduction in any medium, provided the original author(s) and the source are credited.

\section{Appendix: Fractions of Circulating and Trapped Particles}

We start from the elementary volume, $d \Gamma$, in drift phase space $[r, \chi, \varphi ; v, \xi]$

$$
\begin{gathered}
d \Gamma=\sqrt{g} d r d \chi d \varphi v^{2} d v d \xi=Y R^{2} d r d \chi d \varphi v^{2} d v d \xi, \\
\xi=\mathrm{v}_{\|} / \mathrm{v}
\end{gathered}
$$

where $(r, \chi, \varphi)$ are the flux coordinates introduced in [12] and $\sqrt{g}=Y R^{2}$ the corresponding Jacobian. Transforming from $[r, \chi, \varphi ; v, \xi]$ to $[r, \chi, \varphi ; v, \lambda(\xi, r, \chi)]$, where $\lambda$ is normalised magnetic moment,

$$
\begin{aligned}
\lambda(\xi, r, \chi) & =\left(1-\xi^{2}\right) B_{0} / B(r, \chi) \equiv\left(1-\xi^{2}\right) / b(r, \chi), \\
b(r, \chi) & =B(r, \chi) / B_{0},
\end{aligned}
$$

we obtain

$d \lambda=-2 \xi d \xi / b, \quad \xi=\sigma \sqrt{1-\lambda b(r, \chi)}$

allowing the following expression for elementary volume $d \Gamma=0.5 \sum_{\sigma} Y R^{2} d r d \chi d \varphi v^{2} d v b d \lambda / \xi, \quad \sigma= \pm 1$.

Fraction of circulating particles is given by

$$
\begin{gathered}
\eta_{c}(r)=\frac{\Gamma_{c}(r)}{\Gamma(r)}=\frac{\oint d \varphi \oint d \chi \int v^{2} d v \int_{\Delta \xi_{c}} d \xi f Y R^{2} d r}{\oint d \varphi \oint d \chi \int v^{2} d v \int_{-1 \leq \xi \leq 1} d \xi f Y R^{2} d r} \\
=\frac{0.5 \sum_{\sigma} \oint d \varphi \oint d \chi \int v^{2} d v \int_{0 \leq \lambda \leq \lambda_{c}} f Y R^{2} \xi^{-1} d r b d \lambda}{0.5 \sum_{\sigma} \oint d \varphi \oint d \chi \int v^{2} d v \int_{0 \leq \lambda \leq \lambda_{\max }} f Y R^{2} \xi^{-1} d r b d \lambda}
\end{gathered}
$$

Here $r$ is flux surface radius, $\Delta \xi_{\mathrm{c}}$ and $0<\lambda<\lambda_{\mathrm{c}}$ are ranges corresponding to circulating particles in the pitchangle cosine and normalised magnetic moment and $f$-the distribution function. In the case of axisymmetry $(\partial / \partial \varphi=0)$ and of isotropic poloidally homogeneous distribution function $[f=f(r, v)]$ for this fraction we obtain

$$
\begin{aligned}
\eta_{c}(r) & =\frac{\oint d \chi \int_{\Delta \xi_{c}} d \xi Y R^{2}}{\oint d \chi \int_{-1 \leq \xi \leq 1} d \xi Y R^{2}} \\
& =\frac{0.5 \sum_{\sigma} \oint d \chi \int_{0 \leq \lambda \leq \lambda_{c}} \frac{Y R^{2} b d \lambda}{\xi}}{0.5 \sum_{\sigma} \oint d \chi \int_{0 \leq \lambda \leq \lambda_{\max }} \frac{Y R^{2} b d \lambda}{\xi}}=\frac{\left\langle Y R^{2} \Delta \xi_{c}\right\rangle}{2\left\langle Y R^{2}\right\rangle}, \\
\Delta \xi_{c} & \equiv 0.5 \sum_{\sigma} \int_{0 \leq \lambda \leq \lambda_{c}} \frac{b d \lambda}{\xi}
\end{aligned}
$$

where $\langle\ldots\rangle$ means poloidal angle average. In the lowest order of $r_{L} / R$ the $\lambda_{c}$ and $\lambda_{\max }$ are determined by

$$
\lambda_{c}=\frac{1}{b_{\max }}=\frac{1}{b(r, \chi=\pi)}, \quad \lambda_{\max }=\frac{1}{b_{\min }}=\frac{1}{b(r, \chi=0)} .
$$

Correspondingly

$$
\begin{aligned}
\Delta \xi_{c} & =0.5 \sum_{\sigma} \int_{0 \leq \lambda \leq \lambda_{c}} \frac{b d \lambda}{\sqrt{1-\lambda b}}=-\left.\sqrt{1-\lambda b}\right|_{0} ^{\lambda_{c}} \\
& =1-\sqrt{1-\frac{b}{b_{\max }}}
\end{aligned}
$$

and

$$
\begin{gathered}
\eta_{c}(r)=\frac{\left\langle Y R^{2} \Delta \xi_{c}\right\rangle}{2\left\langle Y R^{2}\right\rangle}=1-\eta_{t}(r), \\
\eta_{t}(r)=\frac{\left\langle Y R^{2} \sqrt{1-\frac{b}{b_{\max }}}\right\rangle}{\left\langle Y R^{2}\right\rangle},
\end{gathered}
$$

where $\eta_{\mathrm{t}}$ is the fraction of toroidally trapped particles. In the case of weak poloidal field using 
$\frac{b}{b_{\max }}=\frac{B}{B_{\max }} \cong \frac{R(r, \chi=\pi)}{R(r, \chi)}$

we arrive at

$\eta_{t}(r)=\left\langle\sqrt{1-\frac{R_{\min }}{R}}\right\rangle_{F S}$.

Here

$\langle\ldots\rangle_{F S}=\left\langle Y R^{2}(\ldots)\right\rangle /\left\langle Y R^{2}\right\rangle$

is flux surface average.

\section{References}

1. Polevoi et al., J. Plasma Fusion Res. Ser. 5, 82 (2002)

2. A. Fasoli et al., Nucl. Fusion 47, S264-S284 (2007)

3. V.Y. Goloborod'ko et al., Nucl. Fusion 35, 1523 (1995)

4. V.A. Yavorskij, J.W. Edenstrasser, V.Y. Goloborod'ko, S.N. Reznik, S.J. Zweben, Nucl. Fusion 38(10), 1565-1576 (1998)

5. J.W. Edenstrasser, V.Y. Goloborod'ko, S.N. Reznik, V.A. Yavorskij, S.J. Zweben, Czechoslov. J. Phys. 48(2), 177-182 (1998)

6. V.A. Yavorskij, V.Y. Goloborod'ko, K. Schoepf, J.W. Edenstrasser, S. Kuhn, S.N. Reznik, S.J. Zweben, Contrib. Plasma Phys. 40(3-4), 346-351 (2000)

7. K. Schoepf et al., Kerntechnik 67, 285 (2002)
8. V.A. Yavoskij et al., Nucl. Fusion 43, 1077 (2003)

9. N. Hawkes, V. Yavorskij, Y. Baranov, L. Bertalot, C. Challis, S. Conroy, V. Goloborod`ko, V. Kiptily, K. Schoepf, S. Sharapov, D. Stork, E. Surrey, Contributors to the JET-EFDA work programme. Plasma Phys. Control. Fusion 47, 1475 (2005)

10. V. Yavorskij et al., "Interpretive Modelling of Neutral Particle Fluxes Generated by NBI Ions in JET", paper THW/P7-17 at $23^{\text {rd }}$ IAEA Fusion Energy Conference (Daejeon, Korea, 2010)

11. V. Yavorskij et al., Results of predictive Fokker-Planck modelling of NBI deuterons in ITER. J. Fusion Energ 30(4), 307 (2011)

12. W.W. Heidbrink, G.J. Sadler, Nucl. Fusion 34, 535 (1994)

13. M. Schneider et al., Phys. Plasmas Control. Fusion 47, 2087 (2005)

14. K Tani, M Azumi, Nucl. Fusion 48, 085001 (9 pp) (2008). doi:10.1088/0029-5515/48/8/085001

15. S.P. Hirshman, Phys. Fluids 31, 3150 (1988)

16. T. Ohkawa, Nucl. Fusion 10, 185 (1970)

17. Y.R. Lin-Liu, F.L. Hinton, Phys. Plasmas 4(11), 4179 (1997)

18. V.A. Yavoskij et al., Phys. Plasmas Control. Fusion 43, 249 (2001)

19. G.T.A. Huysmans, J.P. Goedbloed, W. Kerner, in Proceedings CP90 Conference Computational Physics (1991)

20. R.J. Hastie, Astrophys. Space Sci. 256, 177-204 (1998)

21. H. Grad, Phys. Fluids 10, 137 (1967)

22. T. Kurki-Suonio et al., 35th EPS Conference on Plasma Phys. Hersonissos, ECA vol. 32, O-4.036, 9-13 June 2008

23. V. Yavorskij et al., $35^{\text {th }}$ EPS Plasma Physics Conference, Hersonissos, Crete, P1.041 (2008). http://epsppd.epfl.ch/Hersonissos/ pdf/P1_087.pdf 